\title{
Out-of-Focus STEM Method for Imaging Misfit Dislocations in a Hetero Interface
}

\author{
Suhyun $\mathrm{Kim}^{1 *}$, Joong Jung Kim ${ }^{1}$ \\ 1. Memory Analysis Science \& Engineering Group, Samsung Electronics, San \#16 Hwasung-city, 18448, \\ Korea \\ * Corresponding author: suhyun12.kim@ samsung.com
}

Hetero structure of Germanium (Ge) strained to Silicon ( $\mathrm{Si}$ )-substrate has attracted much interest as a ptype channel because of its enhanced hole-mobility [1]. Perfect epitaxial-growth of Ge on the Si exhibits maximum strain field; however, the strain field is locally relaxed around misfit dislocations which are formed at a hetero interface due to the discrepancy in lattice parameters between the two materials. In order to design high-performance electronic device of the hetero structure, it is critical to analysis how the MDs-network forms and distributes at the hetero interface. In the present work, we report on a devised method - the use of out-of-focus in high angle annular dark field-scanning transmission electron microscopy (HAADF-STEM) — for imaging locally-modulated strain field formed by MDs-network.

The $1 \mu$-thick Ge layer was grown on the Si-wafer and a plan-view specimen of the hetero structure of $\mathrm{Ge} / \mathrm{Si}$ was prepared by using a focused ion beam (FIB) as shown in Fig 1(a). For imaging hetero interface between the Ge and $\mathrm{Si}$, the Ge layer was thinned to film thickness of $\sim 5 \mathrm{~nm}$ using FIB milling.

Figure 1(e) represents a schematic view of MDs network formed at the hetero interface of Ge/Si. Periodic arrays of MDs network are typically formed along <110> in-plane directions [2]. In the region between MDs (the $\alpha$-site), the Ge layer is fully strained to the Si-substrate. We attribute the $\beta$-site $\gamma$-site to the regions where the MDs cores formed/crossed each other, respectively. In the regions of $\beta$-site and $\gamma$-site, the strain field is relaxed due to the formation of MDs network.

A high-resolution HAADF image of Fig. 2(a) is acquired when the probe is focused near the top surface of Ge layer, as shown in Fig. 1(b) [2]. The specimen was projected along [001]-zone axis. The bundles of the atomic columns are shown in enlarged view of Fig. 2(d). Two types of features can be seen in the image of Fig. 2(d): Atomic columns exhibiting enhanced contrast (the left side) and those showing reduced contrast (the right side). These features are attributed to the difference in the lattice parameters between two materials ( $\mathrm{Ge}$ and $\mathrm{Si}$ ). The enhanced contrast indicates that the Ge atomic columns are aligned to the $\mathrm{Si}$ atomic columns of the substrate, whereas the reduced contrast indicates that the Ge atomic columns are misaligned to the $\mathrm{Si}$ atomic columns as explained in previous works $[3,4]$.

The image of Fig. 2(b) was recorded by focusing the STEM-probe under the specimen (defocus : -120 $\mathrm{nm}$ ) as schematically shown in Fig. 1(c). In the fully-strained region, the electron channeling is occurred when the beam is under-focused because the convergent electron beams are aligned to each tilted Ge atomic columns as shown in Fig. 1(c). Therefore, in Fig. 2(b), we attributed the brightest contrast to the fully-strained region of the $\alpha$-site.

In addition, we acquired the image by focusing the STEM-probe over the specimen $(+120 \mathrm{~nm})$ and the resulting image is displayed in Fig. 2(c). Surprisingly, the contrast in the image of Fig. 2(c) was reversed with respect to the one of Fig. 2(b). The contrast reversal has been known not to occur in the Z-contrast image because the HAADF detector collects the electrons which are scattered incoherently [5]. Because 
the Z-contrast mechanism alone cannot explain what we observed in Fig. 2 (b) and (c), we name this method "largely defocused probe (LDP)-STEM". We suggest that this methodology can be applied to various hetero structures such as III-V compound semiconductors and the hetero-epitaxy of perovskite.

\section{References:}

[1] Y. Park et al, J. Appl. Phys. 106 (2009), 034304.

[2] S. Kim et al, Appl. Phys. Lett. 105 (2014), 151604.

[3] L. F. Kourkoutis et al, Phys. Rev. B 84, (2011), 075485.

[4] L. F. Kourkoutis et al, Phys. Rev. Lett. 100, (2008), 036101.

[4] S. J. Pennycook, Ultramicroscopy 30, (1989), 58.

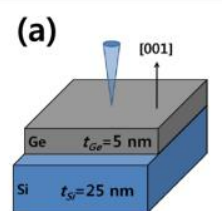

(c)

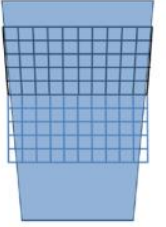

(b)

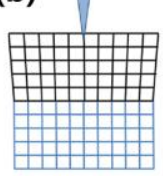

(d)

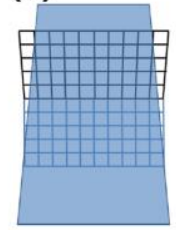

(e)

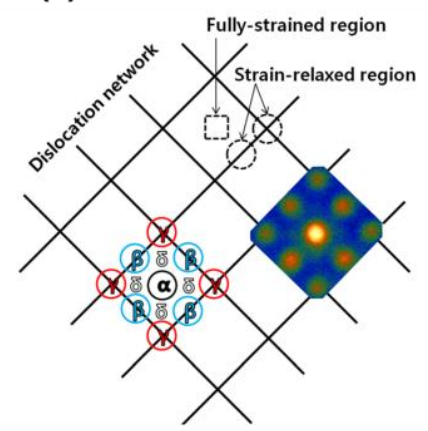

Figure 1. (a) [001] plan-view specimen of Ge/Si hetero structure. (b) Conventional STEM method with a probe focused near the top entrance surface. (c) The out-of-focus method with a probe focused under the specimen and (d) over the specimen. (e) Schematic of dislocations network formed in Ge/Si interface.

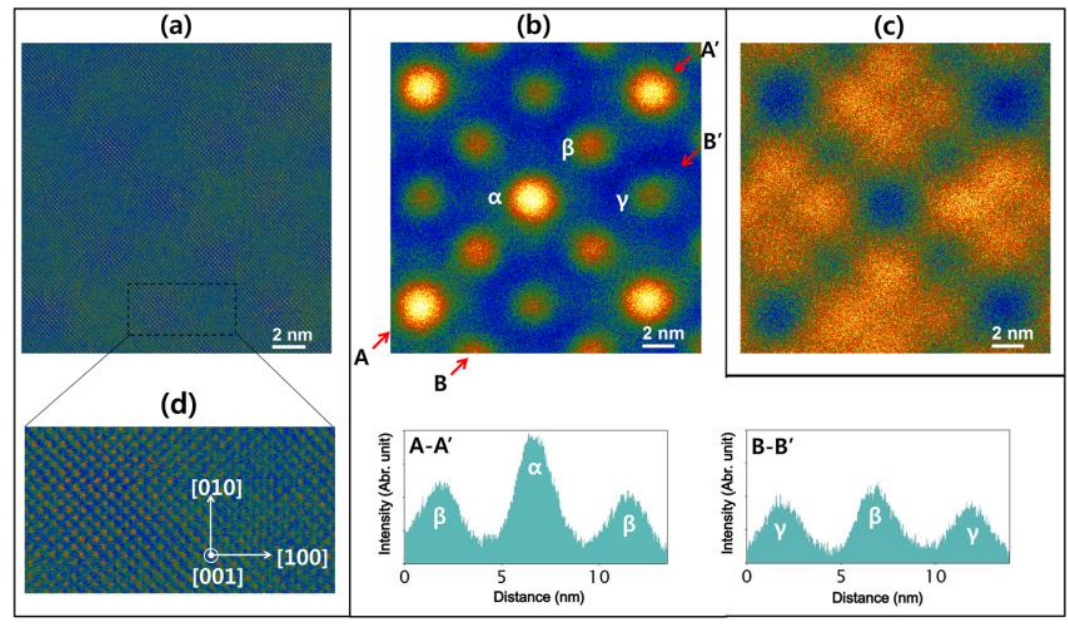

Figure 2. (a) A conventional HAADF image acquired with in-focus condition. (b) Out-of-focused images recorded at under-focus (defocus: $-120 \mathrm{~nm}$ ). The brightest contrast (the $\alpha$-site) is attributed to the electron channeling effect. The relation among the peak intensities is given by the $\alpha$-site $>\beta$-site> $\gamma$-site. (c) Out-of-focused images recorded at over-focus (defocus: $+120 \mathrm{~nm}$ ). The contrast is reversed between these two images of (b) and (c). 